\title{
Assessing rates and contextual predictors of 5-year mortality among HIV-infected and HIV-uninfected individuals following HIV testing in Durban, South Africa
}

Ingrid V. Bassett ${ }^{1,2,3,4^{*}}$ DD, Ai Xu' ${ }^{5}$, Janet Giddy ${ }^{6}$, Laura M. Bogart ${ }^{7}$, Andrew Boulle ${ }^{8,9,10}$, Lucia Millham², Elena Losina $2,3,11,12$ and Robert A. Parker $2,3,4,5$

\begin{abstract}
Background: Little is known about contextual factors that predict long-term mortality following HIV testing in resource-limited settings. We evaluated the impact of contextual factors on 5-year mortality among HIV-infected and HIV-uninfected individuals in Durban, South Africa.

Methods: We used data from the Sizanani trial (NCT01188941) in which adults ( $\geq 18 y$ ) were enrolled prior to HIV testing at 4 outpatient sites. We ascertained vital status via the South African National Population Register. We used random survival forests to identify the most influential predictors of time to death and incorporated these into a Cox model that included age, gender, HIV status, CD4 count, healthcare usage, health facility type, mental health, and self-identified barriers to care (i.e., service delivery, financial, logistical, structural and perceived health).

Results: Among 4816 participants, 39\% were HIV-infected. Median age was 31y and 49\% were female. 380 of 2508 with survival information (15\%) died during median follow-up of 5.8y. For both HIV-infected and HIV-uninfected participants, each additional barrier domain increased the HR of dying by $11 \%$ (HR 1.11,95\% Cl 1.05-1.18). Every 10point increase in mental health score decreased the HR by $7 \%$ (HR 0.93, 95\% Cl 0.89-0.97). The hazard ratio (HR) for death of HIV-infected versus HIV-uninfected varied by age: HR of 6.59 (95\% Cl: 4.79-9.06) at age 20 dropping to a HR of 1.13 (95\% Cl: 0.86-1.48) at age 60.

Conclusions: Independent of serostatus, more self-identified barrier domains and poorer mental health increased mortality risk. Additionally, the impact of HIV on mortality was most pronounced in younger persons. These factors may be used to identify high-risk individuals requiring intensive follow up, regardless of serostatus.
\end{abstract}

Trial registration: Clinical Trials.gov Identifier NCT01188941. Registered 26 August 2010.

Keywords: HIV infection, Mortality, Predictors of mortality, Barriers to care

\section{Background}

South Africa has the largest number of HIV-infected individuals of any country, with over 7 million people diagnosed with HIV and 270,000 new infections in 2016 [1]. People living with HIV (PLWH) who consistently take

\footnotetext{
* Correspondence: ibassett@mgh.harvard.edu

'Division of Infectious Diseases, Massachusetts General Hospital, 100 Cambridge Street, 16th Floor, Boston, MA 02114, USA

${ }^{2}$ Medical Practice Evaluation Center, Massachusetts General Hospital, Boston, MA, USA

Full list of author information is available at the end of the article
}

ART in sub-Saharan Africa can achieve life expectancies similar to those who are HIV-uninfected [2, 3]. However, mortality remains high both before $[4,5]$ and after ART initiation because of inconsistent care [6-10]. Despite 86\% of PLWH in South Africa knowing their HIV status, only $56 \%$ were on ART, and only $45 \%$ were virally suppressed in 2016 [1]. Thus, accurately ascertaining factors contributing to long-term mortality risk following HIV-diagnosis is paramount.

Studies assessing the long-term risk of mortality in persons living with HIV in sub-Saharan Africa have

(c) The Author(s). 2019 Open Access This article is distributed under the terms of the Creative Commons Attribution 4.0 International License (http://creativecommons.org/licenses/by/4.0/), which permits unrestricted use, distribution, and 
focused on age, gender, and CD4 counts to evaluate predictors of risk [11-15]. We and others have found, however, that contextual factors (e.g. barriers to care), emotional health, social support, and competing needs at the time of diagnosis, also likely to have an important effect on survival [16, 17]. In addition, most mortality studies, including those that have examined social and contextual factors, have limited analyses to HIV-infected individuals [7, 18-23]. Little is known about contextual factors that predict long-term mortality in resource-limited settings, for both HIV-infected and HIV-uninfected individuals. By comparing to a concurrently enrolled HIV-uninfected group of individuals, who share similar socioeconomic status, we can further our understanding of what interventions in the outpatient setting may improve outcomes in South Africa regardless of HIV status.

Our objective was to assess contextual predictors of 5year mortality following HIV testing. Research suggests a strong correlation between mental health, especially depression, and mortality among people with HIV [24, 25] and other health conditions [26-28]. Research among PLWH indicates that such associations may be due to a relationship between depression and immune suppression, leading to accelerated disease progression $[25,29,30]$. Moreover, in the US, depression interventions have decreased mortality risk, including for those with chronic diseases, suggesting a causal effect [31-34]. Research also shows strong associations between social support and mortality in general $[35,36]$. Thus, we hypothesized that poor emotional health and social support at HIV diagnosis would be associated with higher 5-year mortality rates and could therefore serve as targets for future interventions.

\section{Methods}

\section{Study setting/design}

This analysis includes data from the Sizanani Trial (NCT01188941), a randomized controlled trial that examined the efficacy of health system navigation and short messaging service (SMS) reminders on linkage to and retention in HIV/TB care. We enrolled adults prior to HIV testing at 4 outpatient sites, 2 hospital outpatient departments (one urban and one semi-rural) and 2 primary health clinics (semi-rural) in Durban, South Africa from August 2010-January 2013. This trial is described in detail elsewhere $[16,37,38]$. Because we did not find efficacy of the intervention with respect to linkage to HIV care, TB treatment completion, or 1-year mortality between study arms, we pooled data from the intervention and control groups into a single cohort in the current study and compared them to HIV-uninfected individuals enrolled concurrently.

\section{Participants}

Adults $\geq 18$ years with unknown HIV status presenting for HIV testing were eligible for enrollment. Study enrollment, consisting of informed consent and a baseline questionnaire, occurred prior to HIV testing. This allowed for assessment of contextual factors, emotional health, and social support prior to knowledge of HIV status.

The study was approved by the McCord Hospital Medical Research Ethics Committee, St. Mary's Hospital Research Ethics Committee, University of KwaZulu- Natal Biomedical Research Ethics Committee, and Partners Institutional Review Board (Protocol 2011-P-001195, Boston, MA).

\section{Data elements}

\section{Demographics and CD4 count data}

We asked participants to provide demographic information including: age, gender, relationship status, and hours worked outside the home. We collected baseline CD4 count data from medical records for those who were HIVinfected; missing data is discussed in the Additional file 1.

\section{Healthcare access}

We assessed healthcare access using four questions that determined how difficult it might be for a patient to reach the site. We collected data on mode of transportation (public, private, or other) and distance to clinic. Transportation variables were grouped into two categories - public transport (bus, taxi) or other (including private and other).

\section{Healthcare utilization}

We assessed self-reported healthcare utilization in the year prior to enrollment, including visits to a community health worker, local clinic, hospital, or private doctor. The total utilization was grouped into three categories: $>5$ times, 3-5 times, 1-2 times, did not use healthcare in prior year. We also asked about number of visits to a traditional healer.

\section{Health behavior}

We asked participants whether they had tested for HIV prior to enrollment. We assessed self-reported competing needs at enrollment, by asking if, in the past 6 months, they had ever gone without healthcare because they needed money for basic needs, or if they had gone without basic needs because they needed money for healthcare [39, 40].

\section{Self-perceived barriers to care}

We assessed self-perceived barriers to healthcare in the 6 months prior to enrollment using a 12-question instrument modified from the ARTAS-II trial [41]. We grouped barriers into 5 domains: 1) concerns about service delivery (waiting time to see a provider, treatment by clinic staff), 
2) financial concerns (ability to afford medication or transportation), 3) perception of personal health (not being sick enough or being too sick), 4) logistical concerns (unable to get out of work, responsibilities to care for others), 5) structural (impaired clinic access due to clinic hours or transportation difficulties, lack of knowledge about where to find care). We created a total number of barriers variable by adding up all barriers in all 5 categories for each participant. We created a similar total number of domains variable by adding up the total number of domains under which a participant indicated they had a barrier.

\section{Emotional health and social support}

We adapted the 5-item Mental Health Inventory screening test and calculated a mental health composite (MHC) score [42]. In addition, we condensed 13 questions about social support into 4 social support scales (emotional/informational, tangible, positive interaction, and affectionate) and calculated the Social Support Index (SSI) from the Medical Outcomes Study [43]. Separately, we averaged each scale and converted to a scale from 0 to 100 . A higher number on the scale indicates better emotional health or social support. An MHC $\leq 52$ qualified as a positive depression screen; an SSI below the sample median (75) qualified as a lack of social support [44].

\section{Outcome ascertainment}

We elicited mortality from the National Population Register, which is estimated to incorporate at least $90 \%$ of deaths nationwide [13, 45]. We used South African ID numbers (SAIDs) obtained at enrollment to match participants to the National Population Register in November 2017; median follow-up was 5.8 years (IQR 5.2-6.5 years).

\section{Statistical analysis}

We provide a summary of our methods here; further details are in the Additional file 1.

Because a substantial fraction of participants were missing SAIDs, we used propensity score (PS) weighting [46] to make the population with SAIDs be representative of the total group. We estimated the probability of having a SAID from a logistic regression model separately for HIV-infected and HIV-uninfected including all available baseline data, and then used inverse probability weighting to make the population with SAIDs more representative of the total population. To avoid potential confounding of contextual factors by HIV-status, we then used an additional propensity score adjustment so that the HIV-infected and HIV-uninfected were similar. We used random survival forests [47] on all covariates listed in Table 1 to inform the development of our survival model. When the number of variables is comparatively large, a random forest is useful for variable selection because it avoids overfitting [48]. We determined variable importance based on permutation importance and variable depth relative to root node (see Additional file 1).

We used a sequential procedure to select the most important variables for the survival model. We first identified the most important of the six domains above to include, and then selected the most important variable(s) in that domain to include in subsequent model construction. Variable selection was based on combining the results from two different statistical approaches. Demographic characteristics were considered the most important category to include, and age was identified as the most important covariate in this category. Gender was selected a priori to be included [49]. Random survival forests were then fitted separately to each of the other five categories of covariates, with age, gender, and HIV status included in all models. The second most important category and important covariates within that category were selected as described above, and the procedure repeated until at least one variable was selected from each category if the category was important. We also evaluated the most important barriers and domains within the self-perceived barriers to care category as detailed in the Additional file 1. Because of the influence of CD4 counts on mortality, we included this in the final model as well. For the 92 HIV-infected participants (8\%) missing CD4, we used multiple imputation based on gender, age, health facility type, healthcare use in the past year, total number of domains, and mental health score CD4 counts. HIV-uninfected participants were assigned a CD4 count of 775 based on the median CD4 count of the general population in rural KwaZulu-Natal [50]. We also assessed how the association of health care utilization with mortality varied over the five-year period by fitting the model for the first third, second third, and last third of deaths separately.

We fitted a propensity score-weighted Cox proportional hazards model to the final set of selected covariates. The likelihood ratio test was used to test for interaction effects between HIV status and other covariates included in the Cox model. We used the integrated area under the curve (AUC) as the measure of accuracy for the Cox models [51].

We describe the association of each variable with death using hazard ratios (HR), 95\% confidence intervals, and $P$-values in the fully adjusted model (incorporating propensity score weighting and $\mathrm{CD} 4$ value imputation), and in simpler models without $C D 4$ value imputation, without propensity score adjustment, and without both to assess the robustness of our conclusions. We used two-tailed $P$-values $<0.05$ as a cut-off for statistical significance. Statistical analyses were performed with SAS version 9.4 (SAS Institute, Cary, $\mathrm{NC}$ ) and "randomForestSRC" in R version 3.4.2 (www. r-project.org) [52]. 
Table 1 Differences between HIV-infected and HIV-uninfected participants at baseline

\begin{tabular}{|c|c|c|c|c|}
\hline & Overall, $n=4816$ & HIV-infected, $n=1897$ & HIV-uninfected, $n=2919$ & $P^{*}$ \\
\hline \multicolumn{5}{|l|}{ Age, yrs } \\
\hline Median (IQR) & $31.0(24-41)$ & $33.0(27-41)$ & $28.0(22-42)$ & $<0.001$ \\
\hline \multicolumn{5}{|l|}{ Sex, n (\%) } \\
\hline Male & $2477[51]$ & $964(51)$ & $1513[52]$ & \multirow[t]{2}{*}{0.491} \\
\hline Female & 2339 [49] & $933(49)$ & $1406[48]$ & \\
\hline \multicolumn{5}{|l|}{ Marital status, n (\%) } \\
\hline Never married & $3738(78)$ & $1535(81)$ & $2203(76)$ & \multirow[t]{3}{*}{$<0.001$} \\
\hline Currently married & $810(17)$ & $265(14)$ & $545(19)$ & \\
\hline Divorce/separated/widowed & $239(5)$ & $85(5)$ & $154(5)$ & \\
\hline \multicolumn{5}{|l|}{ Education, n (\%) } \\
\hline Some high school or greater & $4148(87)$ & $1614(86)$ & $2534(87)$ & \multirow[t]{2}{*}{0.236} \\
\hline Primary school or less & $638(13)$ & $270(14)$ & $368(13)$ & \\
\hline \multicolumn{5}{|l|}{ Mode of transport, n (\%) } \\
\hline Public transport (bus, taxi) & $2283[48]$ & $877(47)$ & $1406[48]$ & \multirow[t]{3}{*}{$<0.001$} \\
\hline Private transport & 1117 [23] & $524(28)$ & $593(20)$ & \\
\hline Other & $1387[29]$ & $484(26)$ & $903(31)$ & \\
\hline \multicolumn{5}{|l|}{ Distance from clinic, n (\%) } \\
\hline Less than $5 \mathrm{~km}$ & $1177[25]$ & $352(19)$ & $825(28)$ & \multirow[t]{2}{*}{$<0.001$} \\
\hline At least 5 km & $3610(75)$ & $1533(81)$ & $2077(72)$ & \\
\hline \multicolumn{5}{|l|}{ Health facility type, n (\%) } \\
\hline Primary health clinics & $1234[26]$ & $404(21)$ & $830(28)$ & \multirow[t]{2}{*}{$<0.001$} \\
\hline Hospital outpatient departments & $3582(74)$ & $1493(79)$ & $2089(72)$ & \\
\hline \multicolumn{5}{|l|}{ Work hours outside home, n (\%) } \\
\hline None & $2697[56]$ & $944(50)$ & $1753[60]$ & \multirow[t]{3}{*}{$<0.001$} \\
\hline Less than $40 \mathrm{~h}$ & $603(13)$ & $318(17)$ & $285(10)$ & \\
\hline $40 \mathrm{~h}$ or more & 1516 [32] & $635(34)$ & $881(30)$ & \\
\hline \multicolumn{5}{|l|}{ Prior HIV testing, $n(\%)$} \\
\hline Yes & 1870 [39] & $464(25)$ & $1406[48]$ & \multirow[t]{2}{*}{$<0.001$} \\
\hline No & $2917(61)$ & $1421(75)$ & $1496[52]$ & \\
\hline \multicolumn{5}{|l|}{ Health care use in prior year, $n(\%)$} \\
\hline None & $715(15)$ & $256(14)$ & $459(16)$ & \multirow[t]{4}{*}{0.006} \\
\hline $1-2$ times & 1499 [31] & $570(30)$ & $929(32)$ & \\
\hline $3-5$ times & $1732[36]$ & $684(36)$ & $1048[36]$ & \\
\hline$>5$ times & $841(18)$ & $375(20)$ & $466(16)$ & \\
\hline \multicolumn{5}{|c|}{ Visit to traditional healer in prior year, $n(\%)$} \\
\hline Yes & $1567[33]$ & $708(38)$ & $859(30)$ & \multirow[t]{2}{*}{$<0.001$} \\
\hline No & $3220(67)$ & $1177(62)$ & $2043(70)$ & \\
\hline \multicolumn{5}{|l|}{ Social support score } \\
\hline Median (IQR) & $75(54-87)$ & $67(50-83)$ & $75(60-90)$ & $<0.001$ \\
\hline \multicolumn{5}{|l|}{ Mental health score } \\
\hline Median (IQR) & $64(56-80)$ & $64(56-76)$ & $68(56-84)$ & $<0.001$ \\
\hline \multicolumn{5}{|l|}{ Reported barriers to healthcare, $n(\%)$} \\
\hline Yes & 1809 [38] & $830(44)$ & $979(34)$ & $<0.001$ \\
\hline No & $3007(62)$ & $1067[56]$ & $1940(66)$ & \\
\hline
\end{tabular}


Table 1 Differences between HIV-infected and HIV-uninfected participants at baseline (Continued)

\begin{tabular}{|c|c|c|c|c|}
\hline & Overall, $n=4816$ & HIV-infected, $n=1897$ & HIV-uninfected, $n=2919$ & $P^{*}$ \\
\hline \multicolumn{5}{|c|}{ Number of barriers for participants reporting barriers } \\
\hline Median (IQR) & $3(2-6)$ & $4(2-6)$ & $3(1-5)$ & $<0.001$ \\
\hline \multicolumn{5}{|c|}{ Number of barrier domains for participants reporting barriers } \\
\hline Median (IQR) & $3(1-4)$ & $3(2-4)$ & $2(1-4)$ & $<0.001$ \\
\hline \multicolumn{5}{|c|}{ Gone without healthcare for basic needs, n (\%) } \\
\hline Yes & $920(19)$ & $414(22)$ & $506(17)$ & $<0.001$ \\
\hline No & $3896(81)$ & $1483(78)$ & $2413(83)$ & \\
\hline \multicolumn{5}{|c|}{ Gone without basic needs for healthcare, n (\%) } \\
\hline Yes & $724(15)$ & $323(17)$ & $401(14)$ & 0.002 \\
\hline No & 4092 (85) & 1574 (83) & 2518 (86) & \\
\hline
\end{tabular}

\section{Results}

\section{Overall cohort characteristics}

There were 4816 enrollees, of whom 1897 (39\%) were HIV-infected (Table 1). The median age of the entire cohort was 31 years (IQR: 24-41); 33 years for HIV-infected individuals and 28 years for those HIV-uninfected $(P<0.001)$. Overall, 2339 (49\%) were female. The median CD4 count for those with HIV was 196 (IQR: 73-352). Most participants, 3738 (78\%), were never married; 2283 (48\%) used public transport to travel to the healthcare site and 3582 (74\%) underwent HIV testing in an hospital outpatient department versus a primary health clinic.

\section{Self-reported barriers to care}

A higher proportion of HIV-infected participants reported one or more barriers to healthcare compared to those HIV-uninfected ( $44 \%$ vs. $34 \% ; P<0.001)$. Among those who reported any barriers, HIV-infected participants also reported more barriers than HIV-uninfected participants: 4 (IQR: $2-6$ ) vs. 3 (IQR: $1-5$; $P<0.001$ ). Likewise, for those who reported any barriers, the total number of barriers spanned more domains for HIV-infected participants than for HIV-uninfected individuals: 3 (IQR: $2-4$ ) vs. 2 (IQR: $1-4 ; P<0.001$ ). 414 (22\%) HIVinfected participants had gone without healthcare for money to spend on basic needs (i.e. food, clothing, housing), whereas only 506 (17\%) HIV-uninfected participants had done so $(P<0.001)$. Similarly, more HIVinfected individuals 323 (17\%) had gone without basic needs for money to spend on healthcare compared to 401 (14\%) HIV-uninfected participants $(P=0.002)$.

Patient perception of not being sick enough to seek care $(1059 ; 22 \%$; Table 2) was the most common individual barrier reported. Uniformly, a higher proportion of participants who tested positive for HIV reported experiencing each type of barrier to care than those who tested negative; all differences were statistically significant. The largest discrepancy between HIV-infected and HIV- uninfected individuals was for waiting too long to see a nurse or doctor: 506 (27\%) of HIV-infected participants reported this barrier, while only 510 (18\%) of HIV-uninfected individuals did $(P<0.001)$.

The most commonly identified barrier domain was patient perception of personal health, with 1247 (26\%) of participants experiencing a barrier in this category. Across barrier domains, service delivery showed the largest difference between HIV-infected and HIV-uninfected participants: $566(30 \%)$ HIV-infected participants identified a service delivery barrier, compared to 586 (20\%) HIV-uninfected participants $(P<0.001)$. HIV-infected individuals experienced significantly higher burdens of self-identified barriers across all domains.

\section{Balance after propensity score adjustment for estimating impact of HIV-infection on mortality}

Of 4816 enrollees, only 1154 of HIV-infected (61\%) and 1354 of HIV-uninfected (46\%) provided valid SAIDs. As shown in Additional file 1: Table S1, there were differences in characteristics between those providing valid SAIDs and those who did not. As shown in Additional file 1: Table S2, initial propensity score weighting of individuals with a valid SAID reduced many of the imbalances between those with a valid SAID and those without valid SAID in both the HIV-infected and HIVuninfected cohorts. A standard propensity score applied to these weighted population reduced imbalances between HIV-infected and HIV-uninfected participants with valid SAID (Additional file 1: Table S3).

\section{Predictors of mortality}

Regardless of HIV status, patients using primary health clinics, as opposed to those using hospital outpatient departments, had reduced mortality risk (HR: 0.51, 95\% CI: 0.38-0.68). A 10-point increase in mental health score decreased the risk of death by 7\% (HR: 0.93; 95\% CI: 0.89-0.97). Participants who used healthcare services in the year before enrollment in the study were at higher 
Table 2 Differences in barriers between HIV-infected and HIV-uninfected participants at baseline

\begin{tabular}{|c|c|c|c|c|}
\hline & Overall, $n=4816$ & HIV-infected, $n=1897$ & HIV-uninfected, $n=2919$ & $P^{*}$ \\
\hline \multicolumn{5}{|l|}{ Barrier domain reported, n (\%) } \\
\hline Service delivery & $1152[24]$ & $566(30)$ & $586(20)$ & $<0.001$ \\
\hline Too long to see nurse/MD & 1016 [21] & $506(27)$ & $510(18)$ & $<0.001$ \\
\hline Not treated with respect & $179(4)$ & $93(5)$ & $86(3)$ & 0.002 \\
\hline Financial & $831(17)$ & $423(22)$ & $408(14)$ & $<0.001$ \\
\hline Cost of transport & $731(15)$ & $377(20)$ & $354(12)$ & $<0.001$ \\
\hline Cost of medication & $760(16)$ & $392(21)$ & $368(13)$ & $<0.001$ \\
\hline Personal Health & $1247[26]$ & $596(31)$ & $651(22)$ & $<0.001$ \\
\hline Not sick enough & 1059 [22] & $503(27)$ & $556(19)$ & $<0.001$ \\
\hline Too sick & $433(9)$ & $244(13)$ & $189(7)$ & $<0.001$ \\
\hline Logistical & $656(14)$ & $334(18)$ & $322(11)$ & $<0.001$ \\
\hline Could not get off work & $382(8)$ & $213(11)$ & $169(6)$ & $<0.001$ \\
\hline Taking care of someone else & $371(8)$ & $182(10)$ & $189(7)$ & $<0.001$ \\
\hline Structural & 1110 [23] & $540(29)$ & $570(20)$ & $<0.001$ \\
\hline Did not know where to find care & $398(8)$ & $181(10)$ & $217(8)$ & 0.033 \\
\hline Difficult hours & $778(16)$ & $402(21)$ & $376(13)$ & $<0.001$ \\
\hline Language barrier & $231(5)$ & $112(6)$ & $119(4)$ & 0.014 \\
\hline Transport & $688(14)$ & 353 (19) & $335(12)$ & $<0.001$ \\
\hline
\end{tabular}

risk of dying, with risk increasing as healthcare use increased (> 5 times, HR: $2.34,95 \%$ CI: $1.75-3.12 ; 3-5$ times, HR: $1.86,95 \%$ CI: $1.42-2.44,1-2$ times, HR: 1.53 , 95\% CI: $1.15-2.02$ compared to no use in the past year). The effect of health care utilization on mortality was reduced over time, showing the most substantial effect in the first third (125 deaths, first 3 months, $P<0.001)$ a smaller but still significant effect in the second third (130 deaths, months $4-21, P<0.001$ ) of deaths and marginal impact in the last third of deaths (125 deaths, month 22 and later, $P=0.07$ ).

There was a significant interaction of HIV-status with age (HR: $0.63,95 \%$ CI: $0.56-0.70, \mathrm{P}<0.001$ for each 10 year increase in age). Each additional 10 years of life increased the risk of death by $94 \%$ for HIV-uninfected participants (HR: $1.94,95 \%$ CI: $1.78-2.11$ ), but only by $22 \%$ (HR: 1.22, 95\% CI: 1.13-1.31; $P<0.001)$ for HIV-infected participants. As shown in Fig. 1, the HR for HIV-infected individuals compared to HIV-uninfected individuals varied from 6.59 (95\% CI: 4.79-9.06) at age 20 down to 1.13 (95\% CI: $0.86-1.48$ ) at age 60. Sensitivity analyses showed that results were robust to the modeling assumptions with HR for HIV-infection ranging from 6.59 to 7.24 at age 20 and 1.13 to 1.29 at age 60 ; model details in Table 3.

After adjusting for the HIV $\mathrm{x}$ age interaction, there was some evidence for an interaction of HIV-status with number of domains, with the mortality risk for HIV-uninfected individuals increasing by $23 \%$ (HR: 1.23 , 95\% CI: 1.14-1.32) for each additional domain, but more slowly for
HIV-infected individuals 7\% (HR: 1.07, 95\% CI: 1.02-1.13; $P=0.002)$ There was also some evidence that the increased hazard for males was lower for HIV-infected men (HR: 1.38, 95\% CI: 1.16-1.65) then for HIV-uninfected men (HR: 2.18, 95\% CI: $1.64-2.89 ; P=0.007)$. Results for HIV-infected and HIV-uninfected individuals separately are reported in Additional file 1: Table S4.

The integrated AUC for the overall Cox regression model was 0.755 . A similar Cox model was fitted separately to the HIV-uninfected and HIV-infected participants with HIV status removed (and CD4 value also removed from the HIV-uninfected population). The model fit was better for HIV-uninfected compared to $\mathrm{H}$ IV-infected participants (AUC 0.837 vs. 0.687).

\section{Discussion}

Among 2503 participants with valid SAIDs and complete data enrolled at 4 outpatient sites in Durban, South Africa between 2010 and 2013, more self-identified barrier domains and poorer mental health increase 5year mortality risk, regardless of HIV status. For every 10-point decrease in mental health score, indicating poorer mental health, 5 -year mortality increased by $7 \%$. For each additional self-identified barrier domain 5 -year mortality increased by $12 \%$. There was some evidence for an interaction between HIV status and risk of 5-year mortality based on number of reported domains. A higher proportion of HIV-infected participants reported self-identified barriers across every domain when compared to HIV-uninfected participants. 


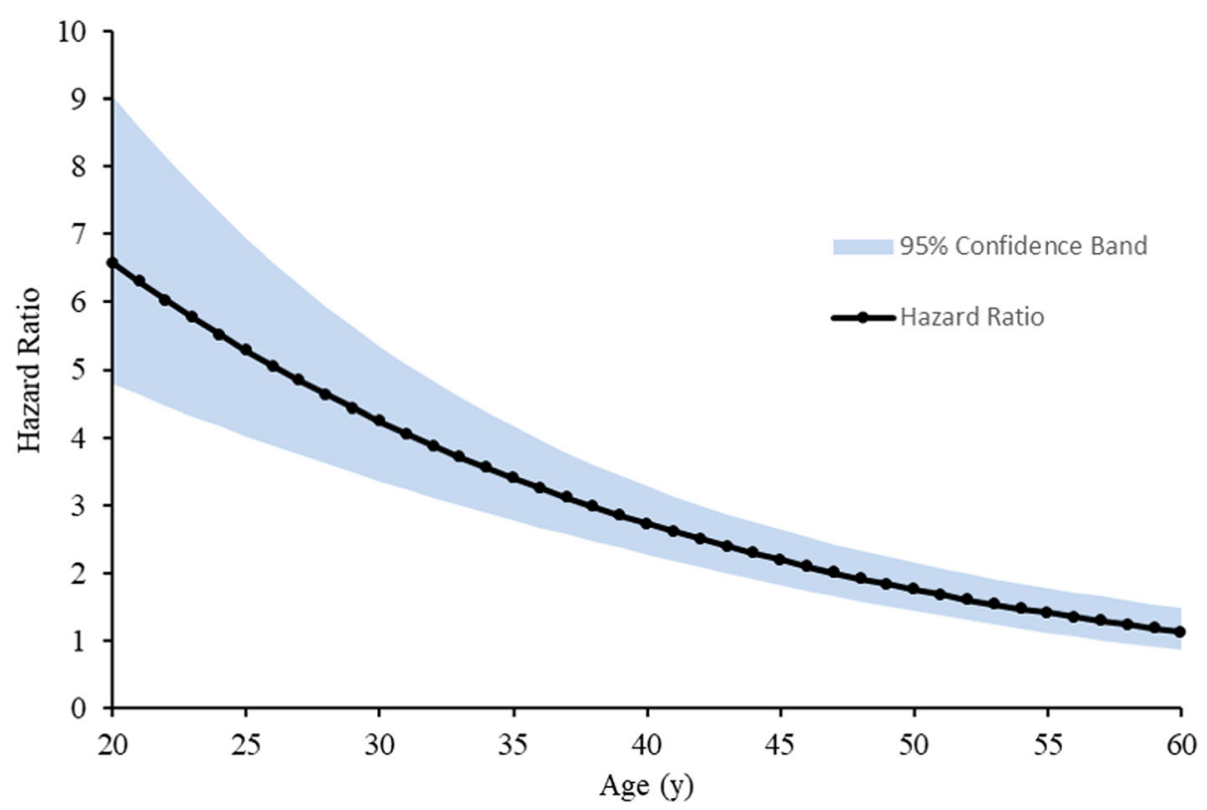

Fig. 1 5-year mortality risk among HIV-infected participants varies by age. Hazard ratio is calculated from the primary model, which includes both two-stage propensity score adjustment and multiple imputation of CD4 values in the HIV-infected population

Additionally, increased healthcare use in the prior year contributed to increased hazard of death for both HIV-infected and HIV-uninfected individuals. Moreover, those who were HIV-infected had a 4-fold increase in hazard of death during follow-up compared to HIV-uninfected participants at age 31 . These results were robust when analyzed across multiple model variations.

This study highlights that barriers to care negatively affect survival regardless of HIV status. HIV-infected individuals reported experiencing significantly more barriers than HIVuninfected individuals. This could be related to HIV-infected participants having fewer resources as significantly more HIV-infected participants also reported more competing needs than their HIV-uninfected counterparts. Among both HIV-infected and HIV-uninfected individuals, perception of personal health, service delivery, and structural barriers were the most frequently reported barrier domains. Within those domains, both HIV-infected and HIV-uninfected participants most commonly reported waiting too long to see a nurse or doctor or not feeling sick enough to seek care as barriers. Recent studies suggest that improvements in clinic operations, including standardizing staff workloads and patient flow, introducing triage, and increasing staff size might help shorten wait times in lowand middle-income settings [53, 54]. We and others have found that in sub-Saharan African settings, participants often feel as though they are not sick enough to seek care $[16,55]$ or avoid seeking care when they do not feel ill for fear that treatment will make them feel worse [56]. Efforts to improve clinic operations and to promote seeking routine care may improve long-term mortality in both HIV-infected and HIV-uninfected individuals.

We found that poor mental health also decreased survival regardless of HIV status. Instituting mental health screening not only during HIV testing but also during routine healthcare appointments could allow providers to identify patients with poor mental health and connect them to additional resources. We and others have found that depressive symptoms are common among HIV-infected patients in sub-Saharan Africa and are correlated with decreased likelihoods of obtaining a CD4 count or taking ART $[57,58]$. In this study, we used the 5-item Mental Health Inventory screening test; this short survey may be feasible to include in routine healthcare visits. On the other hand, social support was not shown to affect 5 -year mortality risk. This may be because we did not measure social support in the form of social integration, which has been shown to be most predictive of mortality $[35,36]$.

Despite continued efforts to diagnose and link individuals to HIV care in South Africa, HIV-infected individuals remain at substantially increased risk for long-term mortality when compared to their HIV-uninfected counterparts at the same study sites and with similar socioeconomic status. A recent study reported similar findings in Botswana comparing a population-based sample of HIV-infected and -uninfected individuals [59]. Other studies have evaluated long-term mortality risk in HIV-infected individuals in subSaharan Africa [11-15], and a few have examined the impact of individual level contextual factors $[16,17]$. Unlike 


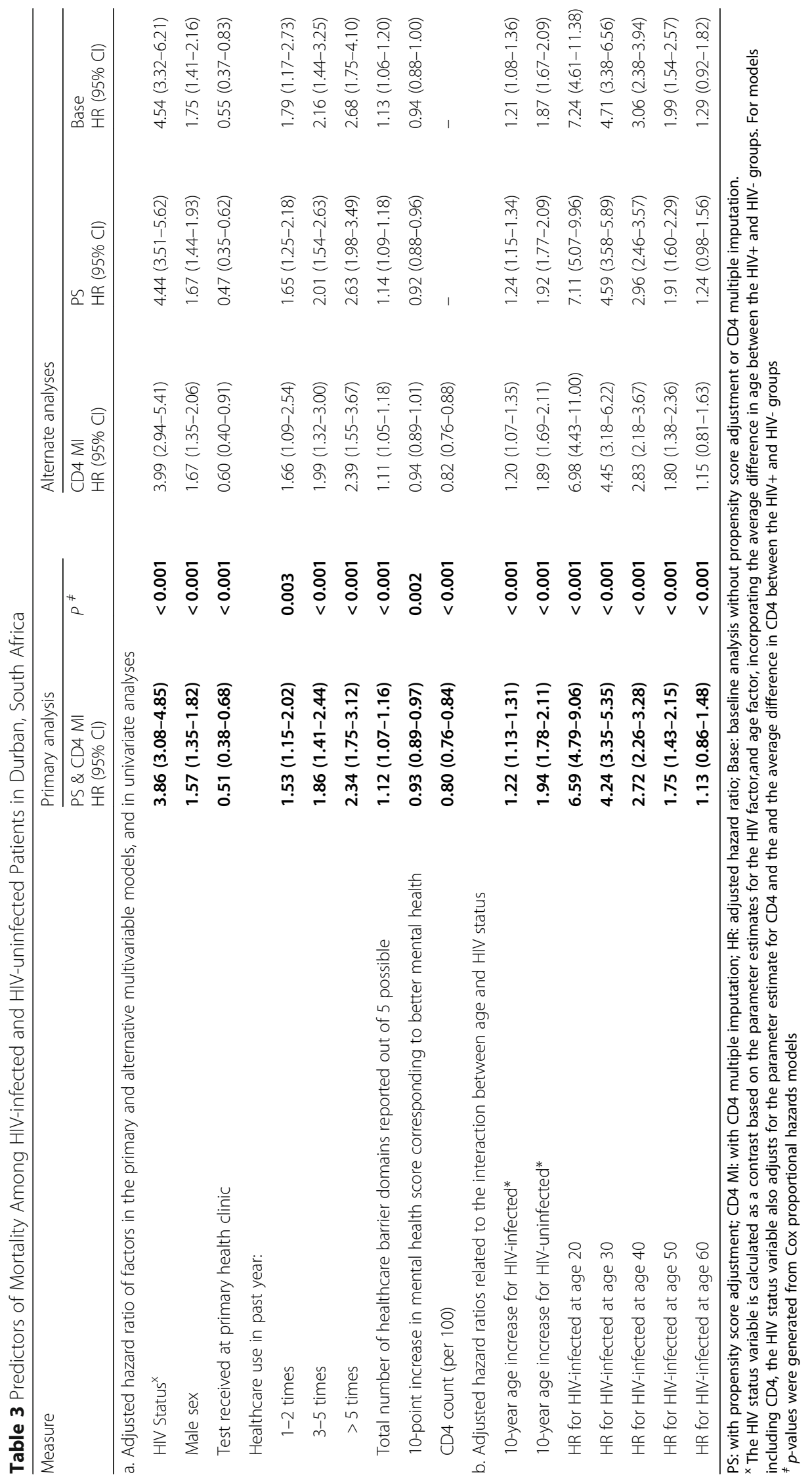


previous studies, however, the present study evaluated HIV-infected and HIV-uninfected individuals and found that some factors previously found to be associated with long-term mortality risk in HIV-infected cohorts, are also risk factors for HIV-uninfected individuals.

This work should be considered in the context of several limitations. We did not adjust for data on ART use in this model; however, CD4 count was an eligibility criterion for starting ART during the study period and was included in the model. We did not collect data on other health-related comorbidities for either HIV-infected or HIV-uninfected participants; it is possible that those who reported more barriers to care may have also had higher rates of comorbid conditions. We also may underestimate the effects of mental health on mortality because we did not consider psychotic symptoms, which have been associated with an even higher relative risk of mortality compared to anxiety and depression [60]. Healthcare use in the prior year may be on the causal pathway to mortality, however, we felt this variable was an important predictor of time to death. We found that the effect of healthcare use in the prior year on mortality was reduced over time and may be a stronger predictor of early mortality than later mortality. The model results did not change qualitatively when this variable was removed (data not shown). In addition, only $52 \%$ of the participants provided a valid SAID number for death registry cross-matching and there were significant differences between those with and without a valid SAID. Though we used propensity scores, we were unable to fully adjust for these differences. Lastly, the methods used to determine predictors of mortality in this study could not be readily used in a clinical setting. In addition to directly addressing those characteristics identified as predictive of mortality, it is necessary to develop predictive instruments, for both HIV-infected and HIV-uninfected individuals, that can be implemented in patient care settings to identify at risk patients.

\section{Conclusions}

HIV infection remains a significant predictor of 5-year mortality in Durban, South Africa. However, additional screening for all patients can be used to help identify at risk individuals who may require additional healthcare interventions. While HIV-infected patients carry a higher burden of self-identified barriers to care than their HIVuninfected counterparts, the effects of those barriers on mortality risk is not significantly different between HIVinfected and HIV-uninfected individuals. Similarly, while HIV-infected participants reported worse mental health than HIV-uninfected participants, poor mental health increased mortality risk for both groups. Interventions are needed that address both clinic-level barriers to care, such as long wait times, as well as patient-level barriers, including efforts to modify beliefs about the risks of HIV treatment, the benefits of seeking care when feeling healthy, and routine mental health monitoring. Such targeted interventions could improve health outcomes for high-risk individuals. Many structural and logistical barriers can be recognized early, i.e. at the first clinic visit, and could identify patients that may require more intensive follow-up. Both alleviation of barriers that pose increased mortality risks and development of tools to identify high-risk patients in clinical settings could significantly improve outcomes for HIV-infected and HIV-uninfected individuals.

\section{Additional file}

Additional file 1: Statistical analysis and supplementary results tables. Variable selection procedure for random forests. Propensity score adjustment procedure. Imputation of CD4 values. Calculated effect of HIV Table S1A and 1B - HIV-infected (1A) and HIV-uninfected (1B) comparing those with and without valid SA ID numbers. Table S2A and 2B show the standardized difference between the overall group (HIV-infected or HIVuninfected) and the group with valid SA ID numbers. (DOCX $74 \mathrm{~kb}$ )

\section{Abbreviations \\ AUC: Area under the curve; HR: Hazard ratio; IQR: Interquartile range; MHC: Mental health composite; PLWH: People living with HIV; SAID: South African Identification Number; SMS: Short messaging service; SSI: Social support index}

\section{Authors' contributions}

All authors have contributed significantly to this work and have read and approved this manuscript. IVB, Principal Investigator of this project, led the design and execution of this study as well as all stages of manuscript writing and preparation. JG and LB also contributed significantly to the design and execution of the study. AB facilitated crossmatch and interpretation of National Population Register data. RAP led all data analysis efforts, while EL also contributed substantially to statistical analysis. AX contributed significantly to data analysis and interpretation. LRIM, the Research Assistant, contributed substantially to manuscript drafting, editing, and revision.

\section{Funding}

This study was funded by the US National Institute of Mental Health R01 MH090326 (IVB) and R01 MH108427 (IVB). This work was also supported by the Weissman Family MGH Research Scholar Award (IVB). The contents of this publication are solely the responsibility of the authors and do not necessarily represent the official views of the US National Institutes of Health. The funder had no role in the design of the study, data collection, analysis, interpretation of data or in writing the manuscript.

\section{Availability of data and materials}

The data supporting these research findings is available upon reasonable request to the corresponding author.

\section{Ethics approval and consent to participate}

The study was approved by the McCord Hospital Medical Research Ethics Committee, St. Mary's Hospital Research Ethics Committee, University of KwaZulu-Natal Biomedical Research Ethics Committee and Partners Institutional Review Board (Protocol 2011-P-001195, Boston, MA). Written informed consent was obtained from all participants.

Consent for publication

Not applicable.

Competing interests

The authors declare that they have no competing interests. 


\section{Author details}

'Division of Infectious Diseases, Massachusetts General Hospital, 100 Cambridge Street, 16th Floor, Boston, MA 02114, USA. ${ }^{2}$ Medical Practice Evaluation Center, Massachusetts General Hospital, Boston, MA, USA. ${ }^{3}$ Harvard Medical School, Boston, MA, USA. ${ }^{4}$ Harvard University Center for AIDS Research, Harvard University, Boston, MA, USA. ${ }^{5}$ Biostatistics Center, Massachusetts General Hospital, Boston, MA, USA. ${ }^{6}$ McCord Hospital, Durban, South Africa. ${ }^{7}$ RAND Corporation, Santa Monica, CA, USA. ${ }^{8}$ Centre for Infectious Diseases, Epidemiology and Research, School of Public Health and Family Medicine, University of Cape Town, Cape Town, South Africa. ${ }^{9}$ Department of Health, Provincial Government of the Western Cape, Cape Town, South Africa. ${ }^{10}$ Wellcome Centre for Infectious Diseases Research in Africa, Institute of Infectious Disease and Molecular Medicine, University of Cape Town, Cape Town, South Africa. ${ }^{11}$ Department of Orthopedic Surgery, Brigham and Women's Hospital, Boston, MA, USA. ${ }^{12}$ Department of Biostatistics, Boston University School of Public Health, Boston, MA, USA.

Received: 17 May 2019 Accepted: 12 August 2019

Published online: 28 August 2019

\section{References}

1. Joint United Nations Programme on HIV/AIDS. UNAIDS Data 2017. Geneva, Switzerland: UNAIDS; 2017.

2. Johnson LF, Mossong J, Dorrington RE, et al. Life expectancies of south African adults starting antiretroviral treatment: collaborative analysis of cohort studies. PLoS Med. 2013;10(4):e1001418.

3. Mills EJ, Bakanda C, Birungi J, et al. Life expectancy of persons receiving combination antiretroviral therapy in low-income countries: a cohort analysis from Uganda. Ann Intern Med. 2011;155(4):209-216.

4. Ingle SM, May M, Uebel K, et al. Outcomes in patients waiting for antiretroviral treatment in the Free State Province, South Africa: prospective linkage study. AIDS. 2010;24(17):2717-2725.

5. Amuron B, Namara G, Birungi J, et al. Mortality and loss-to-follow-up during the pre-treatment period in an antiretroviral therapy programme under normal health service conditions in Uganda. BMC Public Health. 2009;9:290.

6. Bassett IV, Chetty S, Wang B, et al. Loss to follow-up and mortality among HIV-infected people co-infected with TB at ART initiation in Durban, South Africa. J Acquir Immune Defic Syndr. 2012;59(1):25-30.

7. Cornell M, Lessells R, Fox MP, et al. Mortality among adults transferred and lost to follow-up from antiretroviral therapy programmes in South Africa: a multicenter cohort study. J Acquir Immune Defic Syndr. 2014;67(2):e67-e75.

8. Boulle A, Schomaker M, May MT, et al. Mortality in patients with HIV-1 infection starting antiretroviral therapy in South Africa, Europe, or North America: a collaborative analysis of prospective studies. PLoS Med. 2014;11(9):e1001718.

9. Yiannoutsos $C T$, Johnson LF, Boulle $A$, et al. Estimated mortality of adult HIV-infected patients starting treatment with combination antiretroviral therapy. Sex Transm Infect. 2012;88(Suppl 2):i33-43.

10. Braitstein P, Brinkhof MW, Dabis F, et al. Mortality of HIV-1-infected patients in the first year of antiretroviral therapy: comparison between low-income and high-income countries. Lancet. 2006;367(9513):817-824.

11. Cornell M, Johnson LF, Wood R, et al. Twelve-year mortality in adults initiating antiretroviral therapy in South Africa. J Int AIDS Soc. 2017;20(1): 21902.

12. Haas $A D$, Zaniewski $E$, Anderegg $N$, et al. Retention and mortality on antiretroviral therapy in sub-Saharan Africa: collaborative analyses of HIV treatment programmes. J Int AIDS Soc. 2018;21(2).

13. Cornell M, Lessells R, Fox MP, et al. Mortality among adults transferred and lost to follow-up from antiretroviral therapy programmes in South Africa: a multicentre cohort study. J Acquir Immune Defic Syndr. 2014;67(2):e67-e75.

14. Hoffmann CJ, Schomaker M, Fox MP, et al. CD4 count slope and mortality in HIV-infected patients on antiretroviral therapy: multicohort analysis from South Africa. J Acquir Immune Defic Syndr. 2013;63(1):34-41.

15. Fox MP, Shearer K, Maskew M, et al. Treatment outcomes after 7 years of public-sector HIV treatment. AIDS. 2012;26(14):1823-1828.

16. Bassett IV, Coleman SM, Giddy J, et al. Barriers to Care and 1-Year Mortality Among Newly Diagnosed HIV-Infected People in Durban, South Africa. J Acquir Immune Defic Syndr. 2017;74(4):432-438.

17. Mukoswa GM, Charalambous S, Nelson G. The association between social capital and HIV treatment outcomes in South Africa. PLoS One. 2017;12(11):e0184140.
18. Bassett IV, Regan S, Chetty S, et al. Who starts antiretroviral therapy in Durban, South Africa?... not everyone who should. AIDS. 2010;24(Suppl 1):S37-44.

19. Walker AS, Prendergast AJ, Mugyenyi P, et al. Mortality in the year following antiretroviral therapy initiation in HIV-infected adults and children in Uganda and Zimbabwe. Clin Infect Dis. 2012;55(12):1707-1718.

20. Walker AS, Prendergast AJ, Mugyenyi $P$, et al. Mortality in the year following antiretroviral therapy initiation in HIV-infected adults and children in Uganda and Zimbabwe. Clin Infect Dis. 2012;55(12):1707-18.

21. Brinkhof MW, Pujades-Rodriguez M, Egger M. Mortality of patients lost to follow-up in antiretroviral treatment programmes in resource-limited settings: systematic review and meta-analysis. PLoS One. 2009;4(6):e5790.

22. Coetzee D, Hildebrand K, Boulle A, et al. Outcomes after two years of providing antiretroviral treatment in Khayelitsha, South Africa. AIDS. 2004; 18(6):887-895.

23. Flynn AG, Anguzu G, Mubiru F, et al. Socioeconomic position and ten-year survival and virologic outcomes in a Ugandan HIV cohort receiving antiretroviral therapy. PLoS One. 2017;12(12):e0189055.

24. Antelman $G$, Kaaya $S$, Wei $R$, et al. Depressive symptoms increase risk of HIV disease progression and mortality among women in Tanzania. J Acquir Immune Defic Syndr. 2007:44(4):470-477.

25. Ickovics JR, Hamburger ME, Vlahov D, et al. Mortality, cd4 cell count decline, and depressive symptoms among hiv-seropositive women: longitudinal analysis from the hiv epidemiology research study. JAMA. 2001;285(11): 1466-74.

26. Satin JR, Linden W, Phillips MJ. Depression as a predictor of disease progression and mortality in cancer patients. Cancer. 2009;115(22):5349-61.

27. van Dooren FEP, Nefs G, Schram MT, Verhey FRJ, Denollet J, Pouwer F. Depression and risk of mortality in people with diabetes mellitus: a systematic review and meta-analysis. PLoS One. 2013;8(3):e57058.

28. Rutledge T, Reis VA, Linke SE, Greenberg BH, Mills PJ. Depression in heart failure a meta-analytic review of prevalence, intervention effects, and associations with clinical outcomes. J Am Coll Cardiol. 2006;48(8):1527-1537.

29. Burack JH, Barrett DC, Stall RD, Chesney MA, Ekstrand ML, Coates TJ. Depressive symptoms and CD4 lymphocyte decline among HIV-infected men. JAMA. 1993;270(21):2568-2573.

30. Evans DL, Ten Have TR, Douglas SD, et al. Association of depression with viral load, CD8 T lymphocytes, and natural killer cells in women with HIV infection. Am J Psychiatry. 2002;159(10):1752-9.

31. Gallo JJ, Bogner HR, Morales KH, Post EP, Lin JY, Bruce ML. The effect of a primary care practice-based depression intervention on mortality in older adults: a randomized trial. Ann Intern Med. 2007;146(10):689-698.

32. Gallo JJ, Morales KH, Bogner HR, et al. Long term effect of depression care management on mortality in older adults: follow-up of cluster randomized clinical trial in primary care. BMJ. 2013;346:f2570.

33. Bogner HR, Morales KH, Post EP, Bruce ML. Diabetes, depression, and death: a randomized controlled trial of a depression treatment program for older adults based in primary care (PROSPECT). Diabetes Care. 2007;30(12):3005-10.

34. Bogner $\mathrm{HR}$, Joo JH, Hwang S, et al. Does a depression management program decrease mortality in older adults with specific medical conditions in primary care? An exploratory analysis. J Am Geriatr Soc. 2016;64(1):126-31.

35. Holt-Lunstad J, Robles TF, Sbarra DA. Advancing social connection as a public health priority in the United States. Am Psychol. 2017;72(6):517-30.

36. Holt-Lunstad J, Smith TB, Layton JB. Social relationships and mortality risk: a meta-analytic review. PLoS Med. 2010;7(7):e1000316.

37. Bassett IV, Coleman SM, Giddy J, et al. Sizanani: A randomized trial of health system navigators to improve linkage to HIV and TB care in South Africa. J Acquir Immune Defic Syndr. 2016;73(2):154-160.

38. Bassett IV, Giddy J, Chaisson CE, et al. A randomized trial to optimize HIV/TB care in South Africa: design of the Sizanani trial. BMC Infect Dis. 2013;13:390.

39. Rand Corporation. HIV Cost and Services Utilization Study. Available at: http://www.rand.org/health/projects/hcsus/Base/. Accessed 31 October 2014.

40. Cunningham WE, Andersen RM, Katz MH, et al. The impact of competing subsistence needs and barriers on access to medical care for persons with human immunodeficiency virus receiving care in the United States. Med Care. 1999:37(12):1270-81.

41. Craw JA, Gardner LI, Marks G, et al. Brief strengths-based case management promotes entry into HIV medical care: results of the antiretroviral treatment access study-II. J Acquir Immune Defic Syndr. 2008;47(5):597-606.

42. Hays RD, Sherbourne CD, Mazel RM. The RAND 36-item health survey 1.0. Health Econ. 1993;2(3):217-27. 
43. Sherbourne CD, Stewart AL. The MOS social support survey. Soc Sci Med. 1991;32(6):705-14.

44. Holmes WC. A short, psychiatric, case-finding measure for HIV seropositive outpatients: performance characteristics of the 5 -item mental health subscale of the SF-20 in a male, seropositive sample. Med Care. 1998;36(2):237-43.

45. Johnson LF, Dorrington RE, Laubscher R, et al. A comparison of death recording by health centres and civil registration in South Africans receiving antiretroviral treatment. J Int AIDS Soc. 2015;18(1).

46. Rosenbaum $P$, Rubin $D$. The central role of the propensity score in observational studies for causal effects. Biometrika. 1983;70:41-55.

47. Ishwaran H, Kogalur U, Blackstone E, Lauer M. Random survival forests. Ann Appl Stat. 2008;2(3):841-60.

48. Breiman L. Random forests. Mach Learn. 2001;45(1):5-32.

49. Cornell M, Schomaker M, Garone DB, et al. Gender differences in survival among adult patients starting antiretroviral therapy in South Africa: a multicentre cohort study. PLoS Med. 2012;9(9):e1001304.

50. Malaza A, Mossong J, Bärnighausen T, Viljoen J, Newell M-L. Populationbased CD4 counts in a rural area in South Africa with high HIV prevalence and high antiretroviral treatment coverage. PLoS One. 2013;8(7):e70126.

51. Heagerty PJ, Zheng Y. Survival model predictive accuracy and ROC curves. Biometrics. Mar 2005;61(1):92-105.

52. Ishwaran $\mathrm{H}$, Kogalur U. Random Forests for Survival, Regression, and Classification (RF-SRC), R package version 2.6.1. 2018; https://cran.r-project. org/web/packages/randomForestSRC/index.html.

53. Alamo ST, Wagner GJ, Ouma J, et al. Strategies for Optimizing Clinic Efficiency in a Community-Based Antiretroviral Treatment Programme in Uganda. AIDS Behav. 2013;17(1).

54. Wagenaar BH, Gimbel S, Hoek R, et al. Wait and consult times for primary healthcare services in Central Mozambique: a time-motion study. Glob Health Action. 2016;9.

55. Katirayi L, Chadambuka A, Muchedzi A, et al. Echoes of old HIV paradigms: reassessing the problem of engaging men in HIV testing and treatment through women's perspectives. Reprod Health. 2017;14(1):124.

56. Katz IT, Dietrich J, Tshabalala G, et al. Understanding treatment refusal among adults presenting for HIV-testing in Soweto, South Africa: a qualitative study. AIDS Behav. 2015;19(4):704-14.

57. Kinyanda E, Levin J, Nakasujja N, et al. Major Depressive Disorder: Longitudinal Analysis of Impact on Clinical and Behavioral Outcomes in Uganda. J Acquir Immune Defic Syndr. 1 2018;78(2):136-143.

58. Ramirez-Avila L, Regan S, Giddy J, et al. Depressive symptoms and their impact on health-seeking behaviors in newly-diagnosed HIV-infected patients in Durban, South Africa AIDS Behav 2012;16(8):2226-2235.

59. Gaolathe T, Wirth K, Holmes MP, et al. Higher mortality in HIV-infected vs -uninfected adults despite ART, Botswana. CROI; 2018; Boston, Massachusetts.

60. Walker E, McGee R, Druss B. Mortality in mental disorders and global disease burden implications: a systematic review and meta-analysis. JAMA Psychiatry. 2015;72(4):334-41.

\section{Publisher's Note}

Springer Nature remains neutral with regard to jurisdictional claims in published maps and institutional affiliations.

Ready to submit your research? Choose BMC and benefit from:

- fast, convenient online submission

- thorough peer review by experienced researchers in your field

- rapid publication on acceptance

- support for research data, including large and complex data types

- gold Open Access which fosters wider collaboration and increased citations

- maximum visibility for your research: over $100 \mathrm{M}$ website views per year

At BMC, research is always in progress.

Learn more biomedcentral.com/submissions 